\title{
Dosimetric Comparison of the Lung Dose on the Affected Side in Radiotherapy after Breast Cancer Breast-Conserving Therapy Based on Two Subfield Optimization Methods in Monaco Planning System
}

\author{
Zhirui Shan \\ Shenzhen Luohu People's Hospital, Shenzhen 518001, Guangdong, China \\ Email: 838360321@qq.com
}

\begin{abstract}
Objective - To conduct comparison of the quantity difference of different segmentation optimization methods in Monaco planning system on the affected lung in radiotherapy after breast cancer breast-conserving therapy. Methods 10 cases with breast cancer after breast-conserving surgery were selected with the prescription dose of 50GY/25F. In each case, volume modulated arc therapy (VMAT) based double arc distribution mode was adopted, and the same optimization parameters and functions were set accordingly. After completion of flux optimization, automatic weight optimization method and manual weight optimization method were adopted for optimization respectively. When the similar dose distribution of the target volume was obtained and the dose of the organ at risk (OAR) was satisfied, the dose of the affected lung obtained by the two optimization methods was compared. Results - When similar target coverage and other organs at risk met the prescribed conditions, the affected lung received by the manual weight optimization method was significantly lower than that by the automatic weight optimization method, and the results were statistically significant. Conclusion The manual weight optimization method used in VMAT program after breast conserving surgery can effectively reduce the lung volume on the affected side and the probability of radiation pneumonia can be reduced.

Keywords: Monaco planning system, VMAT, automatic weight optimization, manual weight optimization
\end{abstract}

Breast conserving surgery combined with radiotherapy in patients with early breast cancer can improve the local control rate of tumor and the long-term survival rate of patients, which can reduce the mortality rate caused by breast cancer ${ }^{[1]}$. Intensity modulated radiation therapy (IMRT) is endowed with significant advantages in improving the dose distribution in the target area and reducing the dose in the surrounding normal tissue ${ }^{[2]}$. Fixed-field intensity modulation technique fails to meet the conformal requirements of the target volume in radiotherapy for breast cancer patients with complex curvature of the internal breast target region. VMAT can obtain more degrees of freedom by dynamically adjusting the dosage rate, the motion speed of the frame and the shape of the subfield during beam exit. A great number of studies have shown that VMAT technology can improve PTV coverage in breast cancer radiotherapy planning compared with three-dimensional conformal and fixed field intensity modulation techniques ${ }^{[3]}$, which can improve the conformity index and uniformity index of the target volume. However, more efforts are needed to control the organs at risk, especially the dose of the affected lung. The amount of exposure to the affected lung is closely pertinent to the probability of occurrence of radiation pneumonia. How to reduce the dose to the lung while ensuring adequate exposure to the target volume is an important issue that has been discussed in the industry. Based on the above studies, this paper compared the dose of two VMAT optimization methods in the radiotherapy plan after breast conserving surgery for the affected lung, so as to obtain the optimal dose distribution in the target volume and minimize the dose exposure to the lung, so as to provide reference for clinical radiotherapy after early breast cancer surgery ${ }^{[4]}$.

\section{Data and methods}

\subsection{Patient selection}

10 patients with breast cancer after breast-conserving surgery in our unit were randomly selected for the study of treatment plan. The average age of patients for treatment was 43 years old.

\subsection{CT simulation positioning and delineation for target volume}

The patient was placed in a supine position with both hands raised on the position holder to fully expose the mammary gland. After patient's upper body is fixed with body film, a GE 16-slice large-aperture CT with a thickness of $5 \mathrm{~mm}$ was used for enhanced scanning from the mandible to the xiphoid in a calm breathing state. After the scan, the CT images were 
transferred to the Monaco planning system of the Medical Center, and the radiologists were assigned to map the clinical target volume (CTV) and organs at risk (OARS), including the ipsilateral lung, contralateral lung, contralateral breast, heart, spinal cord, and liver. The CTV includes the entire ipsilateral chest wall and the lymph node area around the tumor. The planned target volume (PTV) is uniformly expanded at $5 \mathrm{~mm}$ by the CTV without exceeding the patient's outer contour.

\subsection{Planning design}

Two VMAT optimization plans were designed for each case using the Monaco 5.11 planning system. The prescription dose was $50 \mathrm{GY} / 25 \mathrm{~F}$. The type of accelerator is the infinity linear accelerator of Elekta Limited, and the X-ray energy is $6 \mathrm{MV}$.

To begin with, flux optimization was performed for each case, and all parameters of the two plans were consistent during flux optimization. Monte Carlo algorithm is adopted. Double arcs are used with the same starting angle and ending angle, and two arcs are moved for each arc. The minimum sector angle stands at $30^{\circ}$, the grid spacing is $0.3 \mathrm{~cm}$, the statistical uncertainty is $1.0 \%$, the maximum control point is 120 , and the minimum blade spacing (MLC) is $0.5 \mathrm{~cm}$. The segment width was $0.6 \mathrm{~cm}$, and fluence smoothing was "medium". The algorithm of organ at risk prioritized (constrained) was adopted.

After the flux calculation, it is ensured that the weight of each cost function at risk should not exceed 1 and the weight of the target volume function is not more than 3. Segment optimization is continued. Method one is the automatic weight optimization method, that is, on the basis of the flux optimization of the first step, the segment optimization of the second step is directly carried out. After the completion of the optimization, only the set value of the function is adjusted until the final optimization result is obtained. The second method refers to the manual weight optimization method, that is, the weights of all parameters are set as manual weights during the second step of segment optimization after the completion of the first step of flux optimization. After the result of segment optimization is obtained, the weight of the function is gradually adjusted from low to high without changing the set value of the function, until the final result is obtained.

Both methods ultimately ensure that the coverage of the $95 \%$ target dose is more than $95 \%{ }^{[5]}$, and the coverage of the $105 \%$ prescription dose is less than 5\%. In the organs at risk, the maximum dose for the spinal cord was less than $35 \mathrm{~Gy}$, the average dose for the heart was less than 10Gy, the maximum dose for the healthy breast was less than 6Gy, and the V5 of the healthy lung was less than $15 \%$.

The quantities of V5, V20 and V30 in the affected lung were compared in the results obtained by the two optimization methods in each case. The three parameters were analyzed by statistical analysis software, and $\mathrm{P}<0.05$ was considered statistically significant.

\section{Result analysis}

The results of quantity of the lung on the affected side under the two optimization methods are shown in Table 1. Method 1 shows that the results of V5, V20 and V30 of affected lung obtained by automatic weight optimization algorithm were all higher than those obtained by manual weight optimization algorithm, and the difference was statistically significant $(\mathrm{P}<0.05)$.

Table 1. Two optimization methods were used to calculate the affected lung volume

\begin{tabular}{ccc}
\hline Affected lung & Automatic weighting method & Manual weighting method \\
\hline V5 $(\%)$ & $63.5 \pm 9.4$ & $24.1 \pm 6.6$ \\
V20 $(\%)$ & $29.5 \pm 5.1$ & $18.3 \pm 3.2$ \\
V30 $(\%)$ & $20.3 \pm 3.7$ & 18.6 \\
\hline
\end{tabular}

\section{Discussion}

VMAT method can be used to perform multi-arc rotation irradiation in a short period of time, which has been widely used in the radiotherapy planning after breast conserving surgery. Radiation pneumonia is one of the most important complications in radiotherapy for breast cancer and its probability increases rapidly with the increase of dose and volume of radiation on the lung. Compared with the automatic weight optimization method, the manual weight optimization method can greatly reduce the dose of the affected lung on the basis of ensuring the similar target volume, and to a certain extent, it can reduce the probability of radiation pneumonia.

Monaco planning system adopts Monte Carlo algorithm and biological function calculation method, which is widely used in clinical practice. How to use the planning system to achieve a satisfactory dose distribution is a problem that physicists have been pursuing ${ }^{[6]}$. Mastering the skills of planning system will serves as better facilitate the services in 
the clinical practice. In this study, the differences of the two segment optimization methods in clinical application were compared through data analysis to provide theoretical basis for the study of clinical treatment plan.

Since the dose of radiation exposure to the lung after breast conserving surgery is very important to radiation pneumonia, the dose of radiation exposure to the affected side of the lung was just selected as the object of study, and other OARs were made with limitations on dosage. There are certain limitations. In future studies, the dose of all OARs can be used as a parameter for comparative studies to enrich the research results.

\section{References}

[1] Li Jianbin, Yu Jinming, Xu Min. Progress of radiotherapy after breast conserving surgery. Chinese Journal of Radiation Oncology. 2010; 019(002): 170-175.

[2] Liu Min, Dong Lihua, Wang Huidong, et al. Comparison of parameters between intense-modulated radiotherapy and conventional radiotherapy after breast conserving surgery. Chinese Journal of Gerontology. 2014; 11(11): 14-16.

[3] Bai Han, Hou Yu, Li Wenhui, et al. Design of Volume Modulated Arc Therapy (VMAT) physical plan for whole-breast radiotherapy after breast conserving therapy. Medical Equipment. 2018; 031(001): 9-12.

[4] Wu Qi, Ye Naiyao. Clinical analysis of injury induced by radiotherapy on lung after operation on patients with breast cancer. Medical Science and Technology. 1992: 47-49.

[5] Chen Jihong, Zhang Xiuchun, Lu Jun, Hu Cairong. Dosimetric study of two kinds of planning systems in intense-modulated radiotherapy for breast cancer patients. Medical Equipment. 2016; 11.

[6] Pan Xiang, Yang Yi, Hou Yu, Yuan Meifang. Dosimetric differences of different Monaco-based distribution methods in intensity-modulated radiotherapy after left breast conserving surgery. Journal of Clinical Medicine in Practice. 2021; 1. 